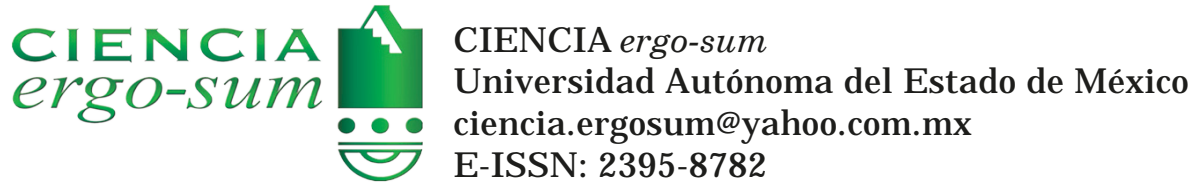

\title{
Determinación de la energía libre superficial del líquido sinovial humano en biomateriales
}

Piña Piña, Gilberto; Rodríguez Lelis, J osé María; Solano Pérez, J usto J uvenal Determinación de la energía libre superficial del líquido sinovial humano en biomateriales

CIENCIA ergo-sum, vol. 28, núm. 3, noviembre 2021-febrero 2022 | e133 Universidad Autónoma del Estado de México, México

Esta obra está bajo una Licencia Creative Commons Atribución-NoComercial-SinDerivar 4.0 Internacional .

Piña Piña, G., Rodríguez Lelis, J . M. y Solano Pérez, J . J . (2021). Determinación de la energía libre superficial del líquido sinovial humano en biomateriales. CIE NCIA ergo-sum,28(3). https://doi.org/10.30878/ces.v28n3a5 


\title{
Determinación de la energía libre superficial del líquido sinovial humano en biomateriales
}

\section{Determination of the surface free energy of human synovial fluid in biomaterials}

\author{
Gilberto Piña Piña \\ Instituto Tecnológico de Toluca, México \\ gpinap@toluca.tecnm.mx \\ (1) http://orcid.org/0000-0002--5198-2660 \\ José Maria Rodríguez Lelis \\ Centro Nacional de Investigación y Desarrollo Tecnológico, México \\ jmlelis@cenidet.tecnm.mx \\ (D) http://orcid.org/0000-0002-8288-8861 \\ Justo Juvenal Solano Pérez \\ Instituto Tecnológico de Veracruz, México \\ jsolanoperez@itver.edu.mx \\ (D) http://orcid.org/0000-0002-7678-5620
}

\author{
Recepción: 07 de marzo de 2019 \\ Aprobación: 24 de septiembre de 2020
}

\begin{abstract}
RESUMEN
Se investigan los resultados de la adherencia entre líquido sinovial humano y dos materiales específicos de implante, politetrafluoretileno (PTFE) y Cobalto-Cromo-Molibdeno (Co-Cr-Mo), a través de la medición de los ángulos de contacto, y también se analiza el comportamiento, que es una función de las interacciones entre líquido y la superficie sólida. Las superficies determinan la relación de esfuerzos cortantes, el coeficiente de fricción y el flujo. La interacción entre el líquido sinovial y los materiales de construcción de la prótesis son poco conocidos. El método utilizado es el de la gota sésil y la evaluación con la ecuación de Young.
\end{abstract}

Palabras Clave: artroplastia de rodilla, líquido sinovial, energías interfaciales, ángulos de contacto, gota sésil.

\begin{abstract}
In this work, the results of adherence between human synovial fluid and two specific implant materials, polytetrafluoroethylene (PTFE) and Cobalt-Chromium-Molybdenum (Co-Cr-Mo), are investigated through the measurement of contact angles. Behavior, which is a function of the interactions between liquid and solid surface, is analyzed. Surfaces determine the shear stress relation, coefficient of friction, and flow. The interaction between synovial fluid and prosthetic construction materials is poorly understood. The method used was the sessile drop, and evaluated with Young's equation.
\end{abstract}

KEYWORDS: Knee arthroplasty, synovial fluid, interfacial energies, contact angles, sessile drop.

\section{INTRODUCCIÓN}

En condiciones fisiológicas normales las articulaciones del cuerpo humano son lubricadas por el líquido sinovial, el cual, además de minimizar el desgaste de las superficies en contacto directo, facilita la dinámica en las articulaciones naturales y protésicas -la sinovia es una solución acuosa electrolítica-. Este fluido lubricante contiene macromoléculas de proteínas, lípidos y proteínas de pequeño tamaño como la albúmina, la gammaglobulina, la transferrina y un $\mathrm{pH}$ entre 7.3 y 7.4; el contenido de proteínas es del orden de $20 \mathrm{mg} / \mathrm{ml}$. Su viscosidad es de 0.1 y 1 [Pa.s]" (Yehia y Duncan, 1975; Dumbleton, 1981; Forster, Fisher y Dowson, 1995; Ahlroos, 2001; Dufour y Pillu, 2006; Sawae, 2009). 
Por otra parte, mientras que la articulación natural está rodeada por una cápsula articular donde la bursa sinovial y la membrana secretan líquido sinovial para lubricarla, la articulación protésica de cadera en la cavidad pseudoarticular se llena de líquido sinovial y con líquido periprotésico alrededor de la articulación; la calidad que posee es algo diferente, pero con concentraciones proteínicas totales relativamente similares (Saari et al., 1993; Mazzucco et al., 2002). La adherencia del lubricante sobre la superficie de los sólidos dependerá de las propiedades de dichos materiales: tipo de estructura atómica, rugosidad y dureza principalmente. La adsorción de los componentes del líquido sinovial sobre los biomateriales tiene un papel fundamental en la lubricación de los implantes (Roba et al., 2007).

Después de una artroplastia de rodilla, la membrana eventualmente se reforma y produce un líquido similar al líquido sinovial llamado pseudolíquido sinovial que lubrica al implante (Heuberger et al., 2005; Mazzucco y Spector, 2004). La interacción del pseudolíquido sinovial con la superficie de rodamiento de la prótesis es de vital importancia después de la cirugía (Roba et al., 2007; Yamada et al., 1999; Mandelin et al., 2005). El conocimiento de la función tribológica después del postoperatorio por artroplastia parcial o total y de menisectomía parcial o total de rodilla se encuentra en estudio (Roba et al., 2007; Dumbledon et al., 1981).

Roba et al. (2007) cuentan con trabajos relacionados con las interacciones de los componentes del pseudolíquido sinovial tales como proteínas y glicoproteínas en materiales usados en implantes. En ellos analiza si los componentes del pseudolíquido sinovial tienen buena adsorción sobre superficies de Co-Cr-Mo y UHWMPE. Sus pruebas de fricción fueron confirmadas en un tribómetro de pin y disco.

Ante la complejidad de la lubricación de las articulaciones cartilaginosas, es necesario considerar los mecanismos que intervienen en las prótesis. En el caso de prótesis de cadera se presentan los mecanismos estudiados por métodos convencionales de ingeniería.

\section{MÉtodos de Mediciones eXPERIMENTALES}

Los métodos experimentales generalmente son medidas de fricción en relación con la curva de Stribeck (gráfica 1). También buscan el espesor de lubricante mediante técnicas de resistividad eléctrica.

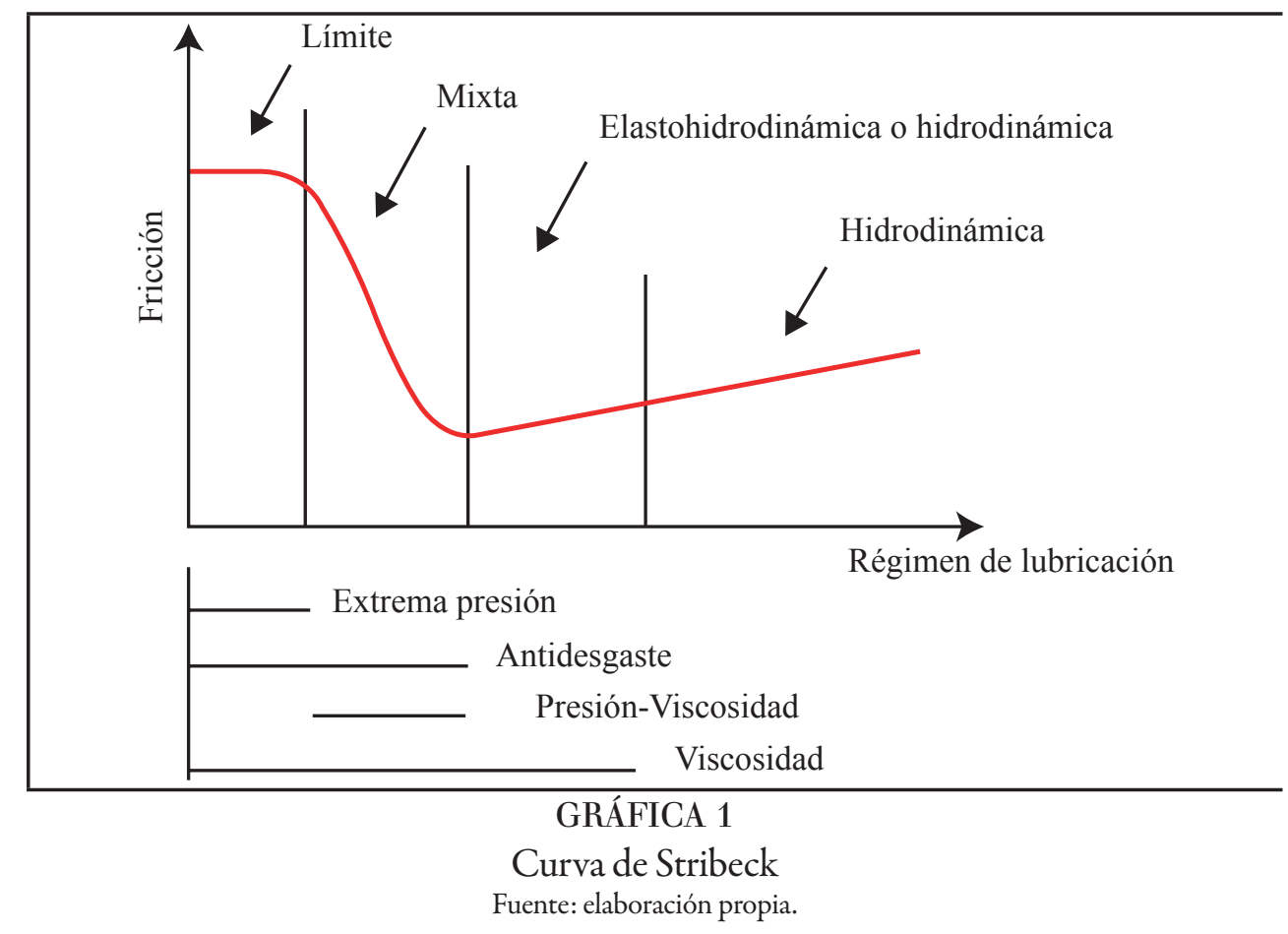


La curva de Stribeck muestra la relación entre el número Hersey, que es un parámetro adimensional de lubricación y del coeficiente de fricción. El número Hersey se define como:

$$
\text { Número de Hersey }=\eta \cdot N / P
$$

Donde $\eta$ es la dinámica de la viscosidad del fluido, $N$ es la velocidad de arrastre del fluido y $P$ es la carga normal en el contacto tribológico. Así, para una viscosidad y una carga dada, la curva de Stribeck muestra cómo la fricción cambia con el aumento de la velocidad. Sobre la base de la progresión típica de la curva de Stribeck, se pueden identificar tres regímenes de lubricación.

\section{MÉtodos PREDICTIVOS-TEÓRICOS}

Se basan en la tasa $\lambda$, que indica el régimen de lubricación. Se define por la siguiente fórmula (Jin, Medley y Dowson, 2003):

$$
\lambda=h_{\text {min }} / \sqrt{\left(R_{a H}\right)^{2}+\left(R_{a C}\right)^{2}}
$$

$h_{\text {min }}=$ espesor mínimo de la película en el implante.

$R_{a} H=$ rugosidad cuadrática media de la superficie de la cabeza femoral.

$R_{a} C=$ rugosidad cuadrática media de la superficie de la copa acetabular en contacto con la cabeza.

La expresión considera la influencia de las propiedades de los materiales, su rugosidad, su esfericidad y el aclaramiento radial de la geometría de la superficie, la calidad y viscosidad del lubricante y las condiciones de funcionamiento (McPherson, 2009).

Los valores de $\lambda$ comprendidos entre 1 y 3 dan lugar a un régimen de lubricación mixto y para valores menores que 1 toda la carga es soportada por los elementos (Smith et al., 2001).

En el régimen de lubricación por capa límite, limítrofe o en película fina, la película sinovial está representada por un simple tapiz de moléculas sobre el que se deslizan las superficies, el cual evita la adhesión de las superficies y permite responder fácilmente a los esfuerzos cortantes que aparecen, además de disminuir su coeficiente de fricción (Ashby, 2008).

Para el régimen elastohidrodinámico, hidrodinámico o en película de lubricante $(\lambda>3)$, se considera que durante el reposo las superficies están en contacto y que el movimiento intercala una película de líquido lubricante que va desde un grosor microscópico (caso de la elastohidrodinámica) hasta un espesor mayor en hidrodinámica (Jin, Medley y Dowson, 2003).

De la misma forma que para la prótesis de cadera, para el caso de la prótesis de la rodilla también es necesario elegir el método más conveniente de lubricación.

Como se empleará un tensiómetro digital comercial para la medida del ángulo de contacto, el método utilizado es el de la gota yacente, que consiste en colocar una gota de líquido sobre la superficie del sólido y estudiar su comportamiento.

\section{TENSIÓN Y ENERGÍA SUPERFICIAL}

La cantidad de energía necesaria para aumentar la superficie por unidad de área $\left(\mathrm{J} \cdot \mathrm{m}^{-2}\right)$ tiene una íntima relación con la tensión superficial de los líquidos, la cual es causada por los efectos de las fuerzas intermoleculares que existen en la interfase y dependerá de la temperatura, del medio que rodea al sistema y de las propiedades del líquido. 
Un líquido tendrá tensión superficial elevada si sus moléculas tienen fuerzas de atracción fuertes. Las moléculas del interior de una masa líquida están sometidas a un campo de fuerzas uniforme que hace que la fuerza resultante sea nula, por lo que se encuentra en equilibrio. La tensión $\gamma_{S L}$ no puede ser medida directamente, ya que se predice con modelos teóricos o al medir simultáneamente el ángulo de contacto $\theta$. Este procedimiento tiende a ser el más común para investigar la mojabilidad de sólidos por líquidos. La mojabilidad, por su parte, se refiere al proceso de interacción entre una superficie sólida y un líquido. Algunos autores expresan este concepto de una manera más técnica: mojabilidad de sólido-líquido es un fenómeno interfacial que a menudo se caracteriza por medir el ángulo de contacto formado entre una gota de líquido y una superficie sólida (Njobuenwu y Nna, 2005). Este fenómeno determina ciertas características de un sólido como la hidrofobicidad y la hidrofilicidad al igual que otros fenómenos de importancia como la tensión superficial.

La figura 2 nos muestra todos los diferentes casos de ángulos de contacto que son posibles de obtenerse en una medición cuando interactúa un sólido y un líquido.

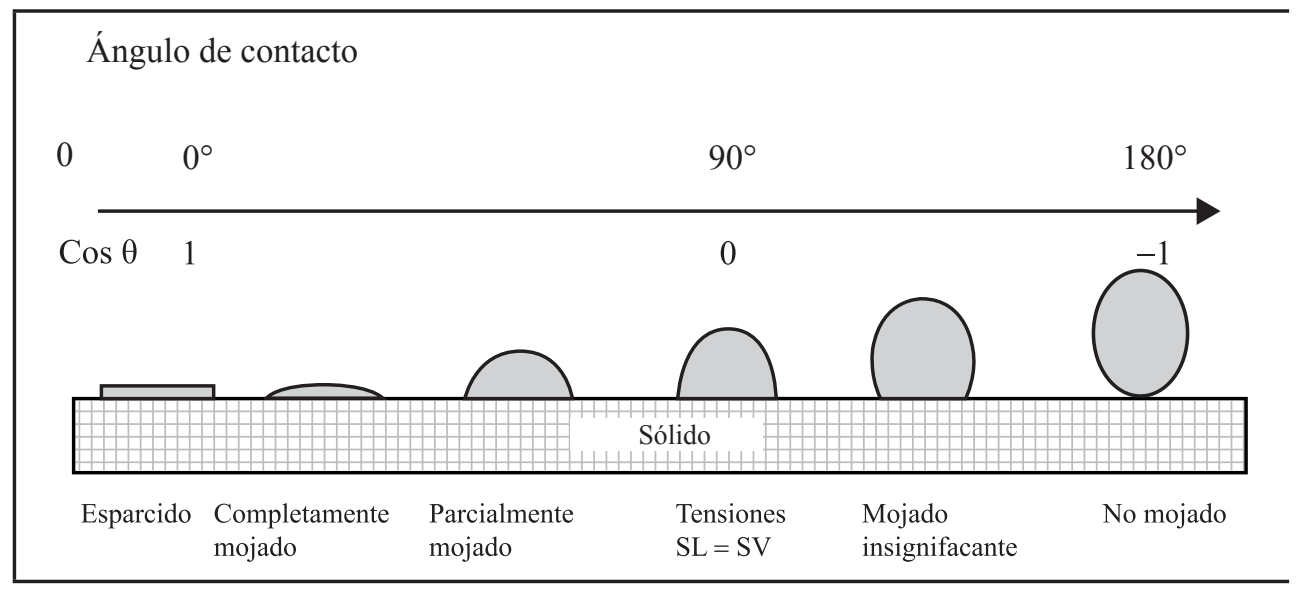

FIGURA 1

Diferentes ángulos de contacto presentes en la interacción líquido-sólido Fuente: elaboración propia con base en Yamada (2009).

\section{Medición del ángulo de Contacto}

El ángulo de contacto entre un líquido y un sólido es aquel que se forma cuando entran en contacto ambos materiales y su valor dependerá de las fuerzas de adherencia entre el sólido y el fluido, además de las fuerzas de cohesión del líquido. En relación con esto, "la mojabilidad es la capacidad de los sólidos para formar interfases con los líquidos. La mojabilidad de un determinado sólido por un líquido viene determinada por el ángulo de contacto $\theta$ " (García Cebadera, 2010) (figura 2).

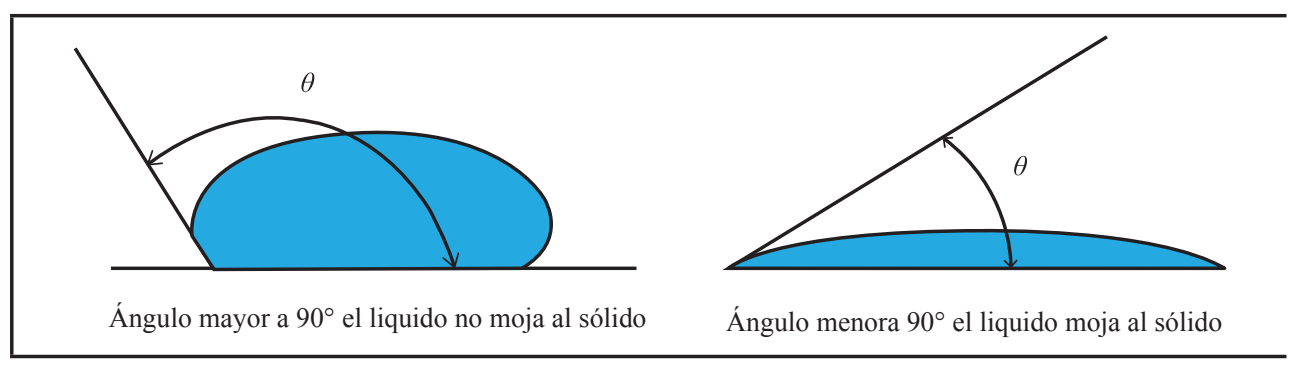

\section{FIGURA 2}

Ángulo de contacto entre sólido y líquido (DataPhyscs)

Fuente: elaboración propia. 
Para realizar las mediciones de los ángulos de contacto se utilizó un equipo comercial modelo DataPhyscs OCA 15EC que consiste en un sistema óptico, una unidad de dosificación automática y un software que permiten medir el ángulo correcto estático y dinámico entre líquidos y sólidos, así como en calcular la energía libre superficial de un sólido y la tensión superficial e interfacial de un líquido con el método de gota pendiente. El equipo también permite determinar la contribución polar dispersiva, la contribución ácido base de las energías de superficie y las tensiones interfaciales. "Cuando se forma un líquido durante la sinterización, la microestructura forma tres intercaras: sólido-vapor, sólido-líquido y líquido-vapor. La mojabilidad describe el equilibrio entre las tres fases y viene determinada por el ángulo de contacto" (Schaffer, Sercombe y Lumley, 2001).

El ángulo de contacto entre el líquido y el sólido se determina al usar la gota yacente. De acuerdo con la figura 3 , se traza una recta tangente de convergencia a las tres fases: $a$ ) fase sólido-líquido, $b$ ) fase sólido-vapor y $c$ ) fase líquido-vapor.

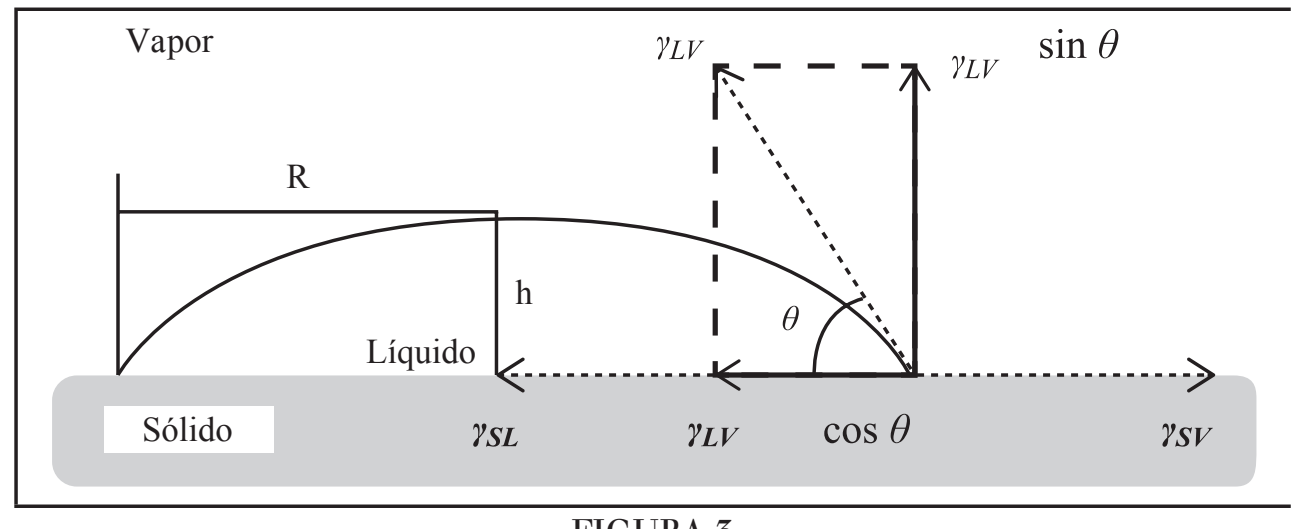

FIGURA 3

Tensión interfacial en las tres fases

Fuente: elaboración propia.

Según la figura 3, $R$ es el radio de la gota y $h$ es su altura. El ángulo de contacto se determina con el contorno de la gota usando la ecuación de Adamson y Gast:

$$
\gamma_{L V} \cos \theta=\gamma_{S V}-\gamma_{S L}
$$

Donde:

$\gamma_{S L}=$ fase sólido-líquido

$\gamma_{S V}=$ fase sólido-vapor

$\gamma_{L V}=$ fase líquido-vapor.

$\gamma_{S V}, \gamma_{S V}$ y $\gamma_{L V}$ son las tensiones interfaciales o energías libres de superficie, las cuales representan la energía libre por unidad de área y $\theta$ es el ángulo de contacto en equilibrio. Al conocer la tensión interfacial del líquido y del sólido, se encuentra el parámetro de difusión $S$ para un caso no equilibrado:

$$
S=\gamma_{S V}-\gamma_{S L}-\gamma_{L V}
$$

$S$ es la energía que se gana al cubrir una unidad de área del sólido con una película plana del líquido lubricante. Si $S$ es negativa, el sólido es cubierto por una película líquida no favorable. Se dice que el líquido se encuentra en un estado de equilibrio si se forma una capa esférica lo bastante pequeña para despreciar los efectos de la gravedad comparada con su capilaridad; esto se caracteriza por el ángulo de contacto en equilibrio denotado por $\theta$. 
Por otro lado, si $S$ tiene un valor positivo se tiene un mojado completo y la gota tiende a propagarse con el tiempo después de ser colocada en la superficie; inicialmente, tomará un ángulo de contacto distinto de 0 hasta llegar a su posición de equilibrio.

La relación de las ecuaciones 3 y 4 se hace al tomar como referencia la figura 3, donde se observa que considerando el radio $R$ y el peso $W$ de la gota, ésta toma el contorno de la superficie del sólido. La interacción entre el líquido y la pared del sólido se expresa con la ecuación siguiente:

$$
\lambda_{L S}=w / 2 \pi R \cos \theta
$$

\section{Métodos y materiales}

Se hace uso de la gota sésil para medir $\gamma_{L V}$ y $\theta$ de manera simultánea. Este método se basa en una gota sésil de líquido que descansa en equilibrio sobre un material sólido horizontal figura 4 (se usa el equipo DataPhyscs descrito). Las imágenes de la gota son obtenidas y se determinan las principales dimensiones de su perfil. La gota es producida mediante un capilar (gota que cae sobre el sólido). La posterior evaluación matemática de las dimensiones de la gota proporciona el valor del ángulo $\theta$ conociendo la densidad del líquido $\gamma_{L V}$. El cálculo se realiza con las expresiones 3,4 y 5 .

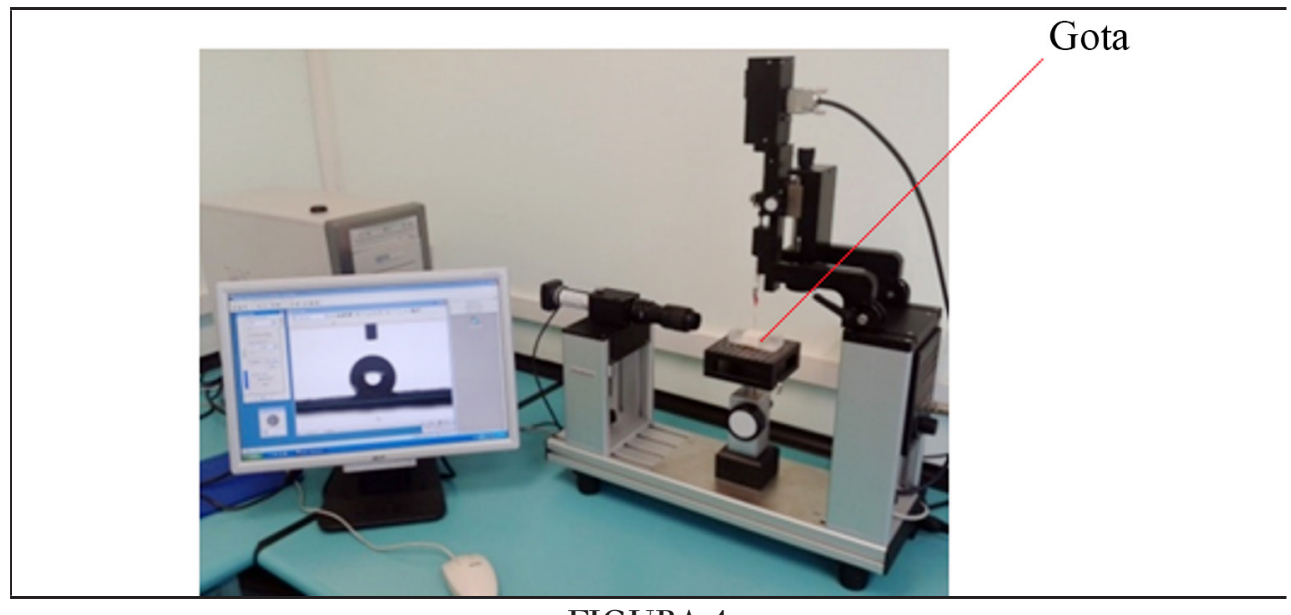

FIGURA 4

Sistema de medición óptico (equipo comercial)

Fuente: elaboración propia.

Para que un estudio experimental sea confiable, el tamaño de muestra debe ser lo suficientemente grande para adquirir un comportamiento normal. En nuestro caso, dicho estudio se validará de forma analítica con la aplicación de las fórmulas ya discutidas.

Por otro lado, también hay que tener cuidado cuando se seleccionen las muestras para evitar la existencia de contaminación que arroje resultados erróneos al momento de recolectar el líquido sinovial; en el caso de los materiales sólidos, éstos deben ser de alta pureza. La presencia de solutos activos en la superficie, incluso en muy bajas concentraciones, puede modificar considerablemente la tensión superficial; por esta razón, los metales se sometieron a una limpieza a los metales a través de métodos de ultrasonido.

Los deportistas de alto rendimiento son personas muy saludables que con frecuencia sufren lesiones en las articulaciones, lo cual facilita la toma de muestras y garantiza la calidad del líquido sinovial.

Se tomaron 50 muestras con un tamaño de cinco elementos cada una de tres pacientes diferentes del sexo masculino; se depositaron 1.5 microlitros de líquido sinovial en la superficie de cada material sólido usando 
una micropipeta. Se midió el ángulo de contacto que forma la gota de líquido sinovial con los materiales sólidos siguientes: fluorocarbonados PTFE, PEUAPM o UHMWPE (ultra-high-molecular-weight-polyethylene) y Co-Cr-Mo. El tamaño de las probetas sólidas fue de $30 \mathrm{~mm}$ x $30 \mathrm{~mm}$ y después de medir todas las muestras se registraron los datos y se aplicó un diseño de experimentos, el cual rectifica los resultados obtenidos. El procedimiento de diseño de experimentos no se muestra en este estudio, ya que es parte de la herramienta estadística empleada.

\section{Resultados Y Discusión}

Como referencia teórica, en la figura 5 se observa que la mojabilidad es buena para $\theta_{1}$ comparada con $\theta_{2}$, donde $\theta_{1}<\theta_{2}$. El ángulo de contacto depende de la diferencia entre las energías de cada fase; la expansión del líquido sobre un sólido es de carácter cinético, el cual se asocia con el mojado e implica una disminución de la energía superficial del sistema.

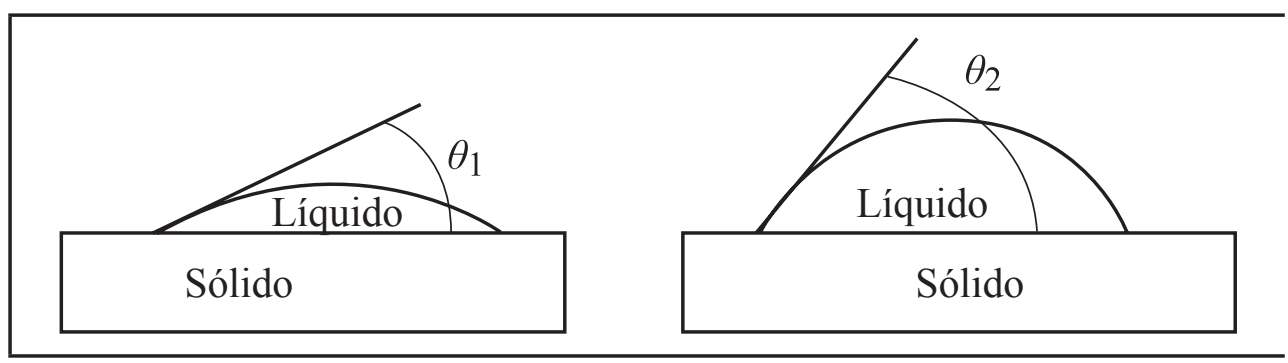

FIGURA 5

Grados de mojabilidad en función del ángulo de contacto Fuente: elaboración propia.

Cuando el líquido se extiende sobre la superficie sólida, su comportamiento dependerá de varios factores tales como su cristalográfica, el estado de la superficie y los contaminantes principalmente.

La figura 6 muestra las gotas medidas sobre los tres materiales estudiados, que son las más utilizadas en la fabricación tanto de prótesis de rodilla como de prótesis de cadera: a) gota de líquido sinovial-PTFE, $b$ ) gota de líquido sinovial-UHMWPE y c) gota de líquido sinovial- Co-Cr-Mo respectivamente. Los resultados se resumen en el cuadro1. Tanto los materiales fluorocarbonados (PTFE) como los polietilenos de ultra alto peso molecular (UHMWPE) reportan ángulos mayores de $90^{\circ}$, los cuales están asociados con un aumento de las energías superficiales y se dice que poseen propiedades hidrofóbicas. Los materiales metálicos (Co-Cr-Mo) dan ángulos mucho menores a $90^{\circ}$, generalmente alrededor de los $60^{\circ}$; este ángulo indica una disminución de la energía superficial del sistema y se habla de una propiedad hidrofílica.

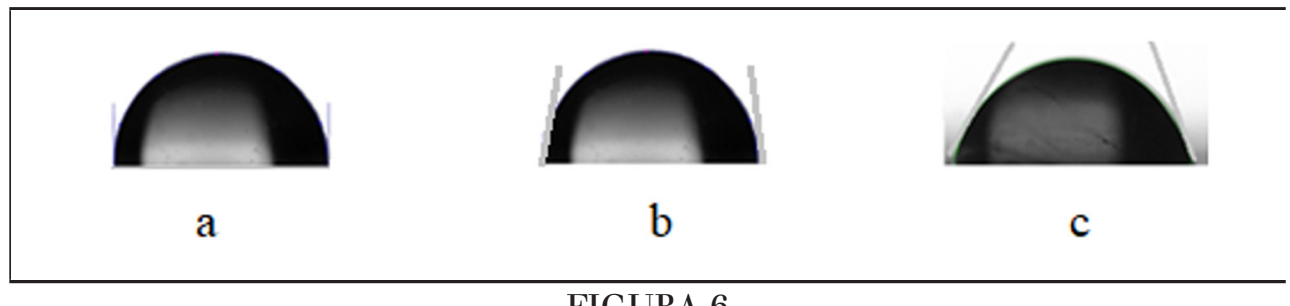

FIGURA 6

a) Gota de líquido sinovial sobre PTEF, $b$ ) Gota de líquido sinovial sobre UHMWPE y c) Gota de líquido sinovial sobre Co-Cr-Mo

Fuente: elaboración propia. 


\section{CUADRO 1}

Resultados de los ángulos de contacto y energía de adhesión entre el líquido sinovial procedente de tres pacientes diferentes hombres y tres biomateriales diferentes

\begin{tabular}{|lcc|}
\hline Paciente 1 & $\theta$ & $\gamma_{S L}$ \\
Materiales & $92^{\circ}$ & $-0.0665 \mathrm{Nm}^{-1}$ \\
PTFE & $58^{\circ}$ & $0.007458 \mathrm{Nm}^{-1}$ \\
Co-Cr-Mo & $90^{\circ}$ & $-0.0060 \mathrm{Nm}^{-1}$ \\
UHMWPE & & \\
\hline Paciente 2 & $\boldsymbol{\theta}$ & $\gamma_{S L}$ \\
\hline Materiales & $94^{\circ}$ & $-0.0680 \mathrm{Nm}^{-1}$ \\
PTFE & $62^{\circ}$ & $0.007458 \mathrm{Nm}^{-1}$ \\
Co-Cr-Mo & $92^{\circ}$ & $-0.0660 \mathrm{Nm}^{-1}$ \\
UHMWPE & & \\
Paciente 3 & $\boldsymbol{\theta}$ & $\gamma_{S L}$ \\
Materiales & $90^{\circ}$ & $-0.0651 \mathrm{Nm}^{-1}$ \\
PTFE & $60^{\circ}$ & $0.007458 \mathrm{Nm}^{-1}$ \\
Co-Cr-Mo & $88^{\circ}$ & $-0.0465 \mathrm{Nm}^{-1}$ \\
UHMWPE & &
\end{tabular}

Fuente: elaboración propia.

La energía superficial se fundamenta en las fuerzas interatómicas, por lo que parámetros como el calor de vaporización, la dureza, el módulo elástico y la temperatura de fusión son indicadores aproximados y representativos de la energía superficial. Para que un líquido moje adecuadamente a un sólido, la energía libre total debe reducirse. El signo negativo en el valor de en los sustratos PTFE y UHMWPE indica una elevada energía libre de superficie. En la mayoría de los ensayos existe una pequeña diferencia en el valor de $\vartheta$ entre ambos lados de la imagen (esto se debe al alineamiento del porta muestras).

En el caso del implante de cadera, los resultados se resumen en la cuadro 2.

\section{CUADRO 2}

Estimación teórica de los regímenes de lubricación de implantes de cadera

\begin{tabular}{|lccc|}
\hline Por fricción & $\begin{array}{c}\text { Espesor mínimo de película } \\
{[\mathrm{Nm}]}\end{array}$ & $\begin{array}{c}\text { Rugosidad media de cada } \\
\text { superficie en contacto [Nm] }\end{array}$ & $\begin{array}{c}\text { Tasa } \\
\text { UHMWPE-metal }\end{array}$ \\
$\begin{array}{llc}\text { Metal-metal } \\
\text { Cerámica-cerámica }\end{array}$ & 38 & $55-95$ & $\begin{array}{c}\text { Película límite } \\
\end{array}$ \\
& 26 & 5 & $1.1-2.4$ \\
Mixto & 3.2 & Película lubricante \\
\hline
\end{tabular}

Fuente: elaboración propia.

En la cuadro 2 se muestran los valores obtenidos para diferentes regímenes de lubricación en función de los tres pares de fricción. El ideal de lubricación es el de película lubricante con el valor de $\lambda$ más alto posible. Las prótesis de cadera metal-polímero operan en régimen de lubricación límite o mixto, lo que ha llevado al diseño de diámetros reducidos de la cabeza femoral tanto como sea posible para reducir al mínimo la fricción y el desgaste.

Por su parte, las prótesis metal-metal bien diseñadas operan en el régimen de lubricación mixta a hidrodinámica 
con gran parte de la carga en apoyos de películas elastohidrodinámicas y su diseño tribológico da como consecuencia un desgaste bajo. Por último, en las prótesis cerámica-cerámica tenemos el régimen de película lubricante, lo que explica su bajo nivel de desgaste a parte de su alta resistencia al mismo por su dureza.

\section{Conclusiones}

De los resultados de las mediciones de los ángulos de contacto y del cálculo de la tensión se obtienen las conclusiones siguientes:

a) El ángulo de contacto depende de la diferencia entre las energías de cada fase y no de sus valores absolutos, esto es, si se conoce el valor de la energía superficial, no implica necesariamente el conocimiento de cómo será la mojabilidad.

b) El aumento de la viscosidad del líquido sinovial mejora las propiedades tribológicas en los pares artificiales utilizados en la fabricación de artroplastias articulares. El líquido articular presente en artroplastias fallidas no parece ser el idóneo, pues el hecho de tener una viscosidad muy baja influye negativamente en sus características tribológicas.

c) El líquido sinovial es muy viscoso debido a su alto contenido en ácido hialurónico. Una disminución de la viscosidad se asocia a un desgaste prematuro. La viscosidad puede ser evaluada subjetivamente mediante un examen citológico. Cuando ésta es normal, las células se disponen en líneas paralelas; si está disminuida, la disposición de las células es desordenada.

d) La variación del ángulo de contacto para las tres diferentes muestras de líquido sinovial nos habla de las diferencias individuales de las personas, de las cuales podemos entender por qué algunas personas sufren lesiones de carácter patológico con el tiempo en las articulaciones.

e) Tanto el polietileno de ultra alto peso molecular UHMWPE como los fluorocarbonados PTFE son hidrofóbicos.

f) El UHMWPE tiene mejor resistencia a la abrasión que el PTEF.

\section{AnÁlisis PROSPECTIVO}

Cuando se sustituye alguna articulación del cuerpo humano, surge la pregunta de cuál será el comportamiento del reemplazo articular, de modo que sea funcional para la persona que recibe el implante. Existen otros elementos tales como los tendones, los ligamentos, la cápsula articular y el líquido sinovial que conjuntamente contribuyen al buen funcionamiento de la prótesis. La importancia de considerar un reemplazo de forma sistémica garantizará el éxito del diseño.

\section{REFERENCIA}

Adamson, A. W., \& Gast, A. P., (1997). Physical Chemistry of Surfaces. New York: Wiley-Interscience. Ahlroos, T. (2001). Effect of lubricant on the wear of prosthetic joint materials. Acta Polytechnica Scandinavica: Mechanical Engineering 53, 2-26.

Ashby, M. F. (2008). Materiales para ingeniería I: Introducción a las propiedades, las aplicaciones y el diseño. Barcelona: Editorial Reverté. 
Dufour, M., \& Pillu, M. (2006). Biomecánica funcional: miembros, cabeza y tronco. Madrid: Elsevier-Masson.

Dumbledon, J. (1981). Tribology of natural and artificial joints. Elsevier.

Forster, H., Fisher, J., \& Dowson, D. (1995). The effect of stationary loading on the friction and boundary lubrication of articular cartilage in the mixed lubrication regime. In Lubricantes and lubrication (Tribology series). México: Elsevier

García Cebadera, A. (2010). Estudio del ángulo de contacto y de la mojabilidad a alta temperatura de fases líquidas en la sinterización de metales (tesis de licenciatura). Escuela Politécnica Superior.

Heuberger, M. P., Widmer, M. R., Zobeley, E., Glockshuber, R., \& Spencer N. D. (2005). Protein-mediated boundary lubrication in arthroplasty. Biomaterials, 26(10), 1165-1173.

Jin, Z. M., Medley, J. B., \& Dowson, D. (2003). Fluid film lubrication in artificial hip joints. Tribology Series, 41, 237-256.

Mandelin, J., Lilijestrom, M., Tian-Fangli Ainola, M., Huukkanen, M., \& Kontitinen, Y. (2005). Pseudosynovial fluid from loosened total hip prosthesis induces osteoclast formation. Journal of Biomedical Materials Research. Part B: Applied Biomaterials, 74(1), 582-588.

Mcpherson, E. J. (2009). Cirugía de reconstrucción articular en adultos. Ortopedia y Traumatología. Revisión sistemática ( 5 a ed.). Ámsterdam: Elsevier.

Mazzuco, D. M., \& Spector. (2004). The John Charnley Award Paper. The role of joint fluid in the tribology of total joint arthroplasty. Clinical Orthopaedics and Related Research, 429, 17-32.

Mazzucco, D., Mckinley, G., Scott, R. D., \& Spector, M. (2002). Rheology of joint fluid in total knee arthroplasty patients. Journal of Orthopaedic Research, 20, 1157-1163.

Njobuenwu, D. O., \& Nna, E. (2005). The effect of critical wetting agent concentration on drilling fluids performance. Journal of Scientific and Technical Research, 4, 65-71.

Roba, M., Naka, M. H., Gasser, B., Delfosse, D., Gautier, E., Spencer, N. D., \& Crockett, R. (2007). Investigation of artificial hip joint lubrication. European Cells and Materials, 14.

Saari, H., Santavirta, S., Nordström, D., Paavolainen, P., \& Konttinen, Y. (1993). Hyaluronate in total hip replacement. Jour. Rheum., 20, 87-90.

Schaffer, G. B., Sercombe, T. B., \& Lumley, R. N. (2001). Liquid phase sintering of aluminium alloys. Materials, chemistry and physics, 67(1-3), 85-91.

Sawae, Y. (2009). Effect of physiological factors on wear of UHMWPE for joint prosthesis en Polymer Tribology. London: Imperial College Press.

Yamada, H., Morita, M., Henmi, O., Miyauchi, S., \& Seki, T. (2009). Hyauloronan in sinovial fluid of patients with loose total hip prosthesis. Comparison with hyaluronan in patients with hip osteoarthritis and idiopathic osteonecrosis of femoral head. Archives of Orthopaedic and Trauma Surgery, 120(9), 521-524

Yehia, S. R., Duncan H. (1975). Synovial fluid análisis. Clinical orthopaedics ans Related Researchs, 107, 11-24.

CC BY-NC-ND 